\title{
Extremely Low Frequency Magnetic Field is an Environmental Stress Factor by Exerting Oxidative Stress
}

\author{
Yong Jin Park ${ }^{1}$, Won Joo Park ${ }^{1}$, Sung Hyuk YiM ${ }^{2}$, Seong Jun YANG ${ }^{3}$, Yuan Lu Sun ${ }^{2}$, \\ Eon Sub PARK ${ }^{1 *}$, and Ji Hoon JEONG ${ }^{2 *}$ \\ ${ }^{1}$ Department of Pathology and ${ }^{2}$ Department of Pharmacology, School of Medicine, \\ Chung Ang University, Seoul, Korea and \\ ${ }^{3}$ Cosmetic Evaluation Team, Korean Food and Drug Administration, Seoul, Korea \\ *The last two authors are co-corresponding authors.
}

(Received March 15, 2007; Accepted March 23, 2007)

\begin{abstract}
The previous study reported the biological effect of magnetic field exerted by acting on endocrine and anti-oxidant system. The present study aims to study whether ELF-MF (extremely low frequency magnetic field) affects the physiological endocrine systems such as thyroid and whether ELF-MF affects the defense system against oxidative stress when it alters the function of thyroid. Finally, we correlate the effects of MF on oxidative stress, and adrenal and thyroid with an environmental stress factor. We exposed sham or MF to rats for 5 or 25 days. After the exposure, we determined pain sensitivity, level of TSH, $\mathrm{T}_{3}$ and free $\mathrm{T}_{4}$ in plasma. We also assayed in whole brain, lipid peroxidation, the activity of enzymatic anti-oxidant defense including superoxide dismutase (SOD) and glutathione peroxidase (GPx), and non enzymatic defense such as reduced or oxidized glutathione contents. MF induced the hypersensitivity to thermal stimuli with the reduction of latency. $T_{3}$ and $T_{4}$ levels were also increased by the exposure of MF. In addition, we observed the rise of MDA level in rat brain by MF although the MF did not change superoxide dismutase and glutathione peroxidase activity. The effect of MF on both reduced and oxidized glutathione results in decrease in reduced or oxidized glutathione in whole brain. In every experiment, there was no significant difference in MF influence between short term (5 days) and long term (25 days) exposure. Taken together, MF exposure affects the thyroid hormonal control in brain. The elevated thyroid hormone acts on brain, leading to hyper-utilization of oxygen. This phenomenon may be correlated with oxidative stress resulting from MF exposure. In conclusion, we suggest that MF exposure may be an environmental stress by exerting oxidative stress.
\end{abstract}

Key words $\square$ extremely low frequency magnetic field, oxidative stress, environmental stress

\section{INTRODUCTION}

Electric and magnetic fields produced by electric power systems have recently been added to the list of environmental agents that are a potential threat to public health. A common concern about extremely low frequency magnetic field (ELFMF) is that whole body exposure to the ELF-MF may have long-term health effects. Even if there is an argument about the biological effect of ELF-MF, ELF-MF have been reported to produce a variety of biological effects (Binhi and Savin, 2002),

\footnotetext{
*Corresponding author

Ji Hoon Jeong, Tel: +82-2-820-5688, Fax: +82-2-826-8752, E-mail: jhjeong3@cau.ac.kr

Eon Sub Park, Tel: +82-2-820-5679, Fax: +82-2-826-8752, E-mail: esp@cau.ac.kr
}

interfere with the activity of the brain (Bruner and Harvey, 1998, Ossenkopp and Cain, 1988; Lai et al., 1998) and may generate behavioral and cognitive disturbances (Keck et al., 2001, Trimmel and Schweiger, 1998). In addition, the previous study suggested that ELF-MF could activate stress-activated protein kinase (Sun et al., 2002) and other investigation observed that magnetotherapy affects the cortisol secretion in the circadian profile by decreasing its level at 16:00 a day after 15 applications, whereas magnetostimulation by increasing its level at 12:00 a month after 15 applications, which may suggest its long-term effect on hypothalamic-pituitary axis (Woldanska-Okonska and Czernicki, 2003). These results indicate that ELF-MF may be an environmental stressor in highly exposed environment.

In experimental animals, exposure to uncontrollable stress induces a number of behavioral and biochemical changes that resemble symptoms seen in human depression and other psy- 
chiatric conditions. Some of the physiologic reactions to stress are: muscle tension, rapid heartbeat, sweaty palms, diarrhea or constipation, increased gastric acid, high blood pressure, increased adrenal hormones, exaggerated mental alertness, increased blood sugar, increased blood lipids, dry mouth, increased insulin; increased thyroid hormone and immune changes (Duntas, 2005; Hart et al., 2005; Levick et al., 2005; Bruno et al., 2006; Huffman et al., 2006; Pantos et al., 2006). There is the demonstration that $T_{3}$ levels change rapidly in response to acute stress (Langer et al., 1983a; Langer et al., 1983b; Langer et al., 1983c). Moreover, hypermetabolic state in hyperthyroidism is associated with tissue oxidative injury (Huffman et al., 2006). Available data indicate that hyperthyroid tissues exhibit an increased ROS and RNS production (Venditti and Di Meo, 2006). Acceleration of $\mathrm{O}_{2}$ uptake by $\mathrm{T}_{3}$ leads to an increased generation of reactive $\mathrm{O}_{2}$ and nitrogen species in the liver, with a higher consumption of cellular antioxidants and inactivation of antioxidant enzymes, thus inducing oxidative stress. This redox imbalance is further contributed by an enhanced respiratory burst activity, which may activate redox-sensitive transcription factors (Fernandez et al., 2006). The increased mitochondrial ROS generation is a side effect of the enhanced level of electron carriers, by which hyperthyroid tissues increase their metabolic capacity. Other thyroid hormone-linked biochemical changes increase tissue susceptibility to oxidative challenge, which exacerbates the injury and dysfunction they suffer under stressful conditions.

It is important to note here that with human beings, health problems are created by psychological needs to identify outside causes of diseases and suffering. This often results in idiopathic environmental intolerance, e.g. multiple sensitivities and symptoms relative to air, water, food and living space factors. Among them, MF may be a crucial factor to lead to stress and result in diseases.

The present study aims to investigate whether ELF-MF affects the physiological endocrine systems such as thyroid and adrenal function and whether ELF-MF affects the defense system against oxidative stress when it alters the function of thyroid and adrenal gland. Finally, we correlate the effects of MF on oxidative stress, and adrenal and thyroid with an environmental stress factor.

\section{MATERIALS AND METHODS}

\section{Animals}

Male SD rats (Hanlim, Seoul, Korea) 4-5 weeks of age and weighing $200 \mathrm{~g}$ were used in all experiments. Animals were maintained in a temperature-controlled room $\left(25 \pm 2^{\circ} \mathrm{C}\right)$ and kept on a 12:12 light dark cycle (lights on at $08: 00 \mathrm{~h}$ and off at 20:00). Food and water were available ad libitum and the rats were adapted for 2 weeks to these conditions before the experiment. The experiments were carried out from 14:00 h to $16: 00 \mathrm{~h}$.

\section{Magnetic fields Exposure system}

The $60 \mathrm{~Hz}$ magnetic field was produced by $1 \mathrm{~m}$-square Helmholtz coil with winding embedded in an open wooden rectangular frame. Each coil has 200 turns and was connected in a series connection to $220 \mathrm{~V}$ AC power supply via variable transformer. Each winding was split allowing the current to flow in the same sense through each half of the winding (field aiding). We can modulate the intensity of magnetic field by the transformer. The magnetic field at the center of exposure system was measured by Gaussmeter [Lake shore Model 410], and we choose $10 \mathrm{G}$ for exposing intensity. The set of coils stood on the platform. We put the animal cage without magnetic material at the center.

\section{Measurement of Pain Thresholds}

Pain thresholds were evaluated with the hot plate (UGO basile model DS-37, Italy) as described by Lakin et al. (1981). Rats were placed individually on the hot plate $\left(50 \pm 0.5^{\circ} \mathrm{C}\right)$ after being allowed to adapt to the experimental environment for a minimum of $90 \mathrm{~min}$ (Pang et al., 2001).

\section{Determination of thyroid hormones}

$T_{3}$ and free $T_{4}$ levels in plasma were measured by radio immunoassay. Plasma (0.2-0.4 ml) was kept frozen until onset of homogenization. This plasma was homogenated in a volume methanol at least three times the weight of the tissue. One thousand five hundred to two thousand cpm of outer ring labeled $\left[{ }^{131} \mathrm{I}\right] \mathrm{T}_{3}$ and $\left[{ }^{125} \mathrm{I}\right] \mathrm{T}_{4}$ were added as internal recovery tracers. After adding chloroform in a double amount of methanol and centrifugation (15 $\mathrm{min}, 2000 \mathrm{rpm}$ ), the pellet was re-extracted in a mixture chloroform-methanol (2:1). Back-extraction into an aqueous phase $\left(0.05 \% \mathrm{CaCl}_{2}\right)$ was followed by a re-extraction with a mixture of chloroform-methanol-0.05\% $\mathrm{CaCl}_{2}$ (3:49:48) and this phase was further purified on Bio-Rad AG 1$\mathrm{X} 2$ resin columns. THs were eluted with $70 \%$ acetic acid, evaporated to dryness, and resuspended in RIA buffer. Recoveries of extracted THs usually ranged from 50 to $75 \%$ for $\left[{ }^{131} \mathrm{I}\right] \mathrm{T}_{3}$ and $40-60 \%$ for $\left[{ }^{125} \mathrm{~T}\right] \mathrm{T}_{4}$. Serum TSH hormone levels were measured using TSH IRMA kit. 


\section{TBARS assay}

Lipid peroxidation, which is a marker of oxidative stress, was determined by measuring spectrophotometrically the formation of thiobarbituric acid-reactive substances (TBARS) (Austin et al., 1995). Each brain area was harvested, sonicated in $1 \mathrm{ml}$ of Tris- $\mathrm{HCl}$ buffer ( $\mathrm{pH} 7.0)$. After centrifugation at $600 \times \mathrm{g}$ for $10 \mathrm{~min}$ at $4^{\circ} \mathrm{C}$ (Micro17TR, Hanil, Korea), $0.9 \mathrm{ml}$ of trichloroacetic acid (8\%) was added to $0.3 \mathrm{ml}$ of supernatant. After centrifugation at $10,000 \times \mathrm{g}$ for $5 \mathrm{~min}$ at $4^{\circ} \mathrm{C}, 0.25 \mathrm{ml}$ of TBA (1\%) was added to $1 \mathrm{ml}$ of supernatant and the resulting solution was heated at $100^{\circ} \mathrm{C}$ for $20 \mathrm{~min}$. The tubes were cooled, $2 \mathrm{ml}$ of $\mathrm{n}$-butanol was added and each tubes was vortexed for $90 \mathrm{~s}$. After centrifugation at $3,000 \times \mathrm{g}$ for $5 \mathrm{~min}$ at $4^{\circ} \mathrm{C}$, $1 \mathrm{ml}$ of butanol phase was utilized for TBARS assay at $532 \mathrm{~nm}$ (UV-160A, Shimadzu, Japan) against malondialdehyde (MDA) standards. Results were expressed as nmol/mg protein. Protein assay was determined according to the Bradford method.

\section{Superoxide dismutase activity assay}

A spectrophotometric assay was used to quantify both total $(\mathrm{Cu}, \mathrm{Zn}$ and $\mathrm{Mn})$ SOD activity and Mn SOD activity alone. This assay was also described previously (Oberley and Spitz, 1984). Each protein extract ( $10 \mathrm{~g})$ was assayed and compared with 1 Unit of bovine $\mathrm{Cu}, \mathrm{Zn}$ SOD (Sigma) activity. The assay was carried out for $2 \mathrm{~min}$ after addition of the radical producer, xanthine oxidase. The SOD activity was expressed as percentage inhibition of the formazan produced in control reaction without SOD or protein extracts. $100 \%$ formazan product formation is the amount of Nitro Blue Tetrazolium reduced by radicals formed by xanthine oxidase in $2 \mathrm{~min}$. Assays were performed in triplicate or duplicate.

\section{Glutathione Peroxidase activity assay}

The GPx activity was measured according to the method described by Sinet (1975) using $t$-butyl-HPx as substrate. The hippocampi were homogenized in PBS $0.05 \mathrm{M}(\mathrm{pH}$ 7.4) in buffer solution $(1 \mathrm{mg} / 5 \mathrm{l}$ ). After homogenization, $30 \mathrm{~g}$ of hippocampal protein was added to 5001 of $\mathrm{PBS} \mathrm{pH} 7.0$ containing $10^{-3} \mathrm{M}$ reduced glutathione, 2 units of yeast glutathione reductase (Sigma type III) and $2 \times 10^{-4}$ NADPH. After $10 \mathrm{~min}$ at 37 ${ }^{\circ} \mathrm{C}$, the reaction was initiated by the addition of $t$-butyl-HPx to a final concentration of $10^{-3} \mathrm{M}$, under constant agitation. The oxidation of NADPH was calculated using extinction coefficient for NADPH of $6.22 \times 10^{3}$ at $340 \mathrm{~nm}$ and the reaction was made for $5 \mathrm{~min}$. One enzyme unit was defined as $1 \mathrm{M} \mathrm{NADPH} /$
$\mathrm{mU}$ per $\mathrm{mg}$ of protein.

\section{Reduced and oxidized glutathione}

The following solutions were required to perform the OPA assay: Redox quenching buffer (RQB) $(20 \mathrm{mM} \mathrm{HCl}, 5 \mathrm{mM}$ DTPA, $10 \mathrm{mM}$ ascorbic acid); 5\% TCA in RQB (TCA-RQB); $7.5 \mathrm{mM} N$-ethylmaleimide (NEM) in RQB; $1.0 \mathrm{M} \mathrm{KP}_{\mathrm{i}}$ buffer (pH 7.0); $0.1 \mathrm{M} \mathrm{KP}_{\mathrm{i}}$ buffer (pH 6.9); $100 \mathrm{mM}$ dithionite (DT; sodium hydrosulfite) in RQB; $5.0 \mathrm{mg} / \mathrm{ml}$ OPA in methanol. DT and OPA solutions were prepared immediately before use. Standards were prepared as follows: $0.1 \mathrm{mM}$ GSSG in TCARQB; $0.1 \mathrm{mM}$ GSH in TCA-RQB. (Note: for method development, it was necessary to remove the trace levels of GSSG that were present in freshly prepared GSH solutions. This was accomplished by treating $1.0 \mathrm{ml}$ of a $1.0 \mathrm{mM}$ solution of GSH in TCA-RQB with $25 \mathrm{mg}$ of zinc dust. The solution was mixed, centrifuged, and filtered through a $0.20-\mu \mathrm{m}$ cellulose acetate syringe filter, and diluted to $0.1 \mathrm{mM}$ with TCA-RQB.) The procedures to determine GSH (Protocol R) and GSSG (Protocol O) using OPA are described by Senft et al. (2000). OPAderived fluorescence was measured at 365 -nm excitation (slit width $5 \mathrm{~nm}$ ) and $430 \mathrm{~nm}$ emission (slit width $20 \mathrm{~nm}$ ).

\section{Statistical analysis}

Analysis of variance was used to establish the statistical significance of differences among group. If significant differences among groups were obtained using the analysis of variance, Duncan's multiple range test was used to differentiate differences between groups. Differences were considered significant when $P$ value is less than 0.05 .

\section{RESULTS}

\section{Thermal response by MF}

MF significantly reduced latency in hot plate test when exposed to rat for 5 days or 25 days. There was no significant difference between 5 day and 25 day MF exposure group (Figure 1).

\section{Effect of MF on thyroid stimulating hormone and thyroid hormones}

We evaluated the levels of thyroid stimulating hormone and thyroid hormones in plasma after the rats were exposed to $\mathrm{MF}$ for 5 or 25 days. The exposure of MF did not change the concentration of TSH (Figure 2). In contrast, the levels of $T_{3}$ and free $T_{4}$ were significantly elevated by MF exposure at 5 days, 


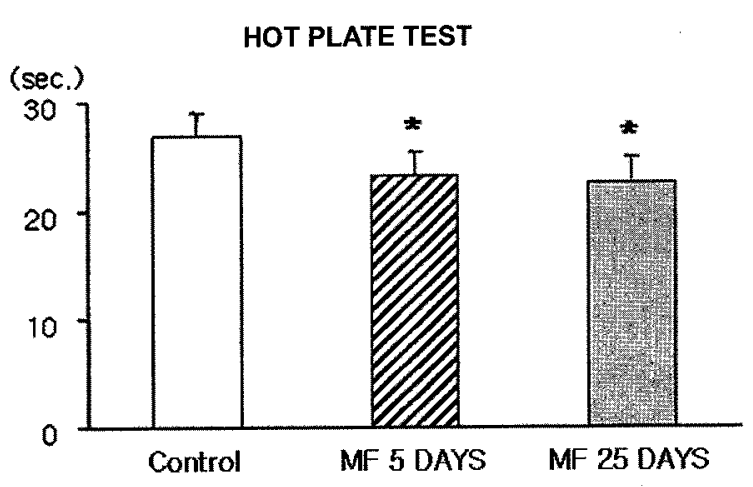

Fig. 1. Animals were exposed to normal condition for 5 or 25 days and tested on the hot plate. Shown are the meanstS.E.M. of the ratio of latency recorded as described in Material and methods. $*(\mathrm{P}<0.05)$ designates significant differences compared to ratio of latency versus control.

but not at 25 days (Figure 3).

\section{Lipid peroxidation by MF exposure}

The influence of MF exposure on free radical production was investigated by measuring the final product of reaction between free radicals and lipids. We used thiobarbituric acid reactive substance assay to confirm the alteration of lipid peroxidation in whole brain (Fig. 4). We observed the significant rise of MDA level in rat brain, suggesting that lipid- peroxidation occurred after MF exposure.

\section{Enzymatic defense systems}

Superoxide dismutase and glutathione peroxidase activity was assayed to evaluate the effect of MF exposure on enzymatic defense systems against oxidative stress. The enzyme

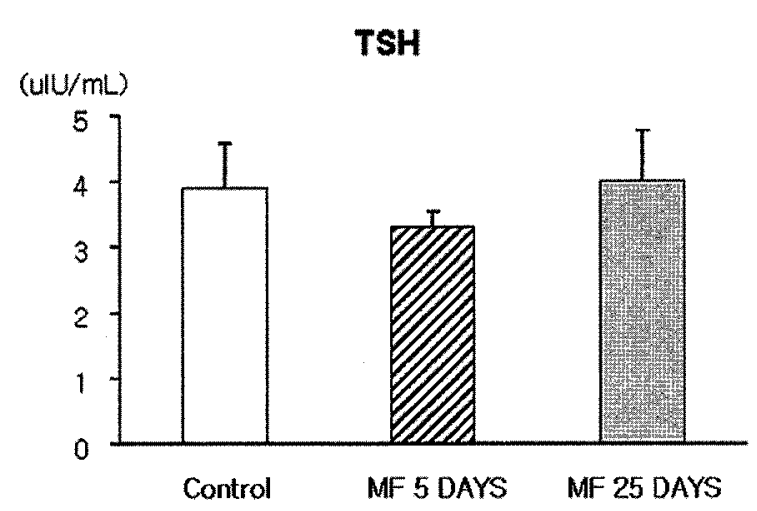

Fig. 2. Animals were exposed to normal condition for 5 or 25 days and the plasma levels of TSH were determined in immunoradioassay. Shown are the means \pm S.E.M. of the ratio of latency recorded as described in Material and methods.

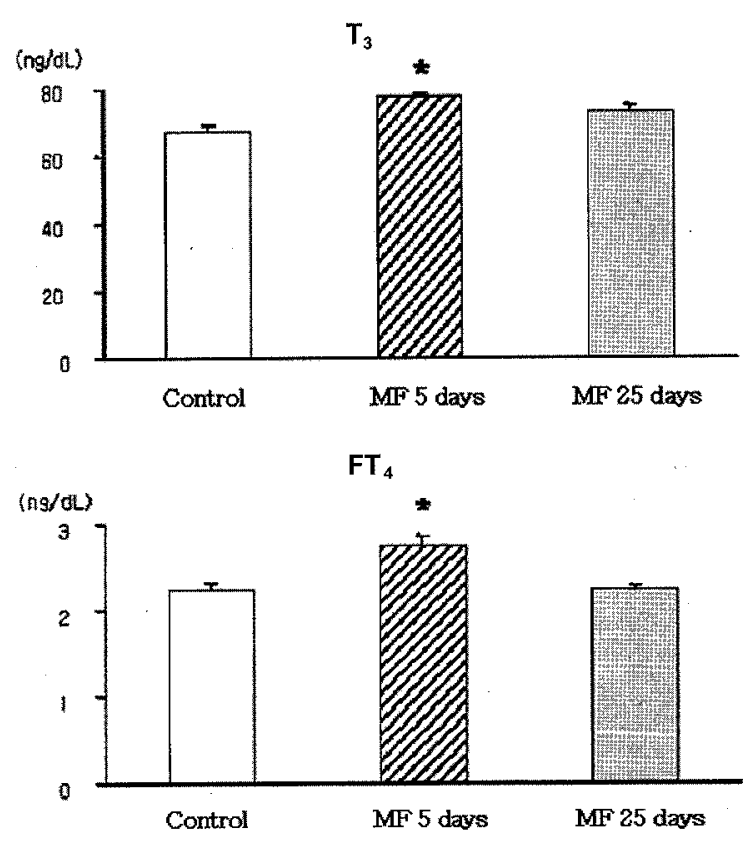

Fig. 3. Animals were exposed to normal condition for 5 or 25 days and the plasma levels of $T_{3}$ and free $T_{4}$ were determined in immunoradioassay. Shown are the means \pm S.E.M. of the ratio of latency recorded as described in Material and methods. $*(\mathrm{P}<0.05)$ designates significant differences versus control.

activity assays were conducted with whole brain exposed to MF for 5 or 25 days. The 5 or 25 day MF exposure to rat did not change both of the enzyme activity (Figure 5,6).

\section{Reduced and oxidized glutathione}

We observed the alteration of reduced and oxidized glu-

\section{MDA CONCENTRATION}

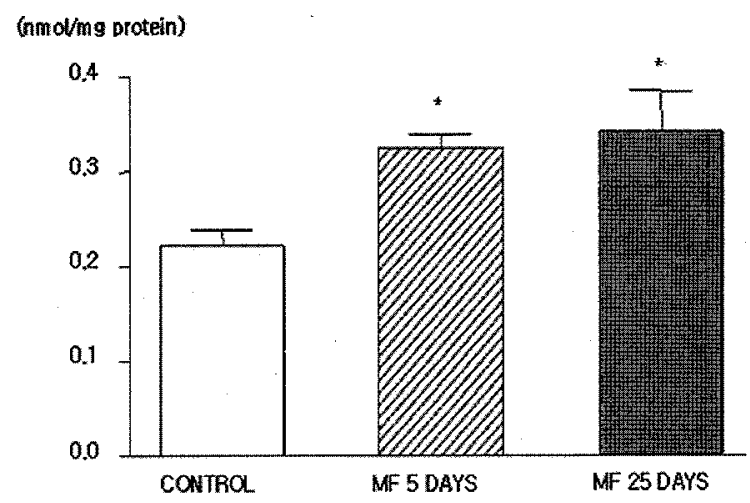

Fig. 4. Animals were exposed to normal condition for 5 or 25 days and the MDA levels were determined in the whole brain. Shown are the means \pm S.E.M. of the ratio of latency recorded as described in Material and methods. * $(\mathrm{P}<0.05)$ designates significant differences versus control. 


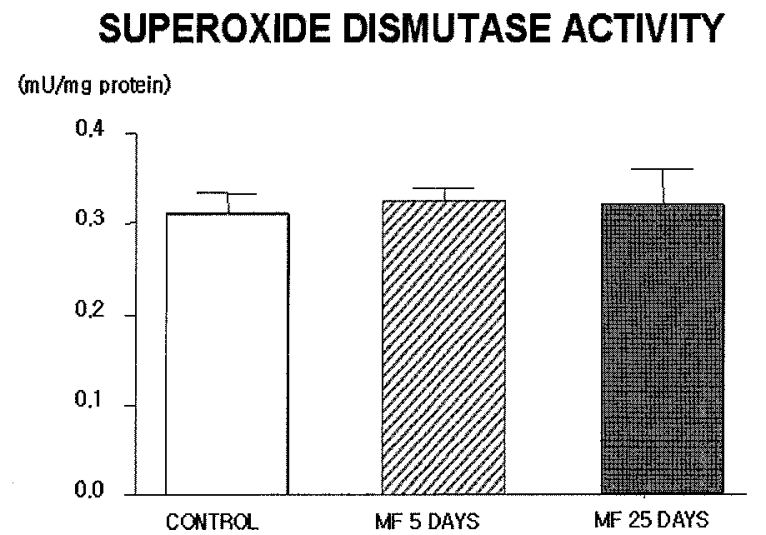

Fig. 5. Animals were exposed to normal condition for 5 or 25 days and the superoxide dismutase activity were determined in the whole brain. Shown are the means \pm S.E.M. of the ratio of latency recorded as described in Material and methods.

tathione acting as non-enzymatic defense components against free radicals in biology. Assays on reduced glutathione resulted in decrease in reduced glutathione in whole brain exposed to MF for 5 days or 25 (Figure 7). In particular, the reduction in whole brain was significantly different from sham controls. Oxidized glutathione was also affected by $\mathrm{MF}$ exposure and reduced significantly in whole brain (Figure 8).

\section{DISCUSSION}

In the present study, MF exposure to rats induced hyper-thermal response and increased thyroid hormones. Although cortisol levels responding to MF exposure were not accurately measured (data not shown), we observed that the cortisol levels were affected by $\mathrm{MF}$ exposure, indicating a possible response to stress. The elevation of thyroid hormones and the alteration of cortisol are a marker of biological anti-stress response against stressful environmental stimuli (Walpita et al., 2006).

The present results suggests that the environment of the MF in this study has an influence in the biological response against stressors and that MF exposure might act as a environmental stressor, which are evidenced by the increase in thyroid hormone and the alteration of cortisol. While cortisol is an important and helpful part of the body's response to stress, it's important that the body's relaxation response to be activated so the body's functions can return to normal. Unfortunately, in our current high-stress culture, the body's stress response is activated so often that functioning often doesn't have a chance to return to normal, producing chronic stress. Psychosocial and somatic stressors induce a complex but uniform neuroendo-

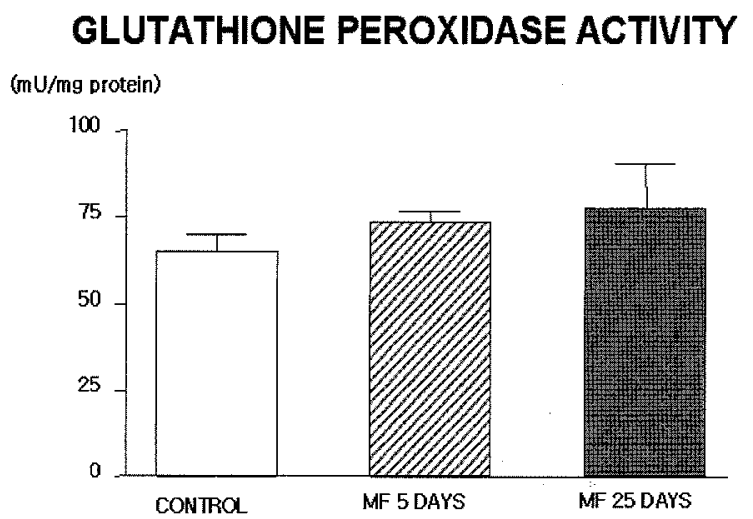

Fig. 6. Animals were exposed to normal condition for 5 or 25 days and the glutathione peroxidase activity were determined in the whole brain. Shown are the means \pm S.E.M. of the ratio of latency recorded as described in Material and methods.

crine activation in rats which is characterized by heavy adrenocorticat secretion and the cessation of the secretion of somatropic hormone (Mazure et al., 1997; Walker et al., 2001). The increase in secretion of the thyroid hormones with this type of effect on the organism of rats may be produced by acute or chronic stress (Langer et al., 1983b; Langer et al., 1983c).

The rise of $T_{3}$ and $T_{4}$ by $M F$ exposure significantly increased the lipid peroxidation in the whole brain. Moreover, the exposure of MF decreased the level of GSH and GSSG, resulting in higher levels of GSSG and GSSG/GSH ratio. An investigation demonstrated that thyroid hormone-induced oxidative stress led to higher levels of GSSG and GSSG/GSH ratio (Varghese et al., 2001; Ishii and Tanizawa, 2006). In contrast, the expo-

\section{REDUCED GLUTATHIONE LEVEL}

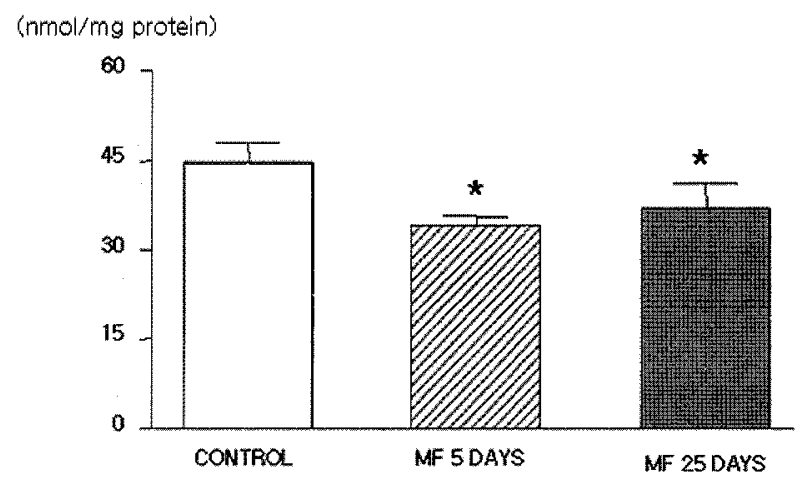

Fig. 7. Animals were exposed to normal condition for 5 or 25 days and the reduced glutathione were determined in the whole brain. Shown are the means \pm S.E.M. of the ratio of latency recorded as described in Material and methods. ${ }^{*}(\mathrm{P}<0.05)$ designates significant differences versus control. 


\section{OXYDIZED GLUTATHIONE LEVEL}
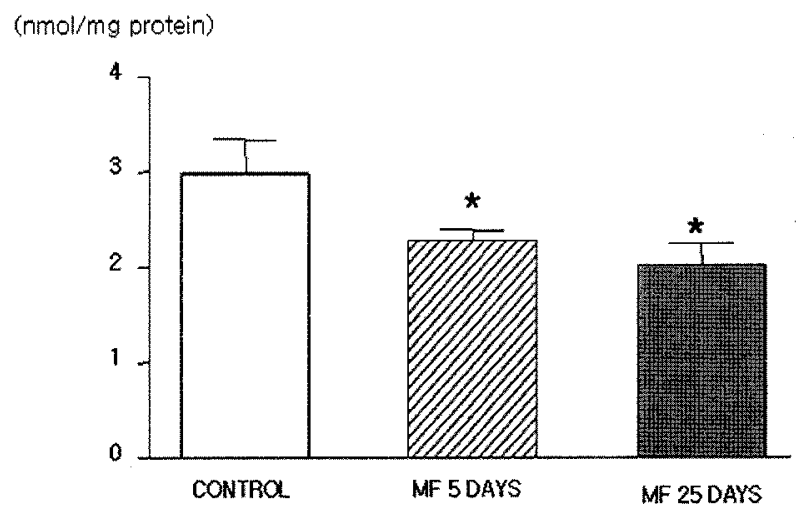

Fig. 8. Animals were exposed to normal condition for 5 or 25 days and the oxydized glutathione were determined in the whole brain. Shown are the means \pm S.E.M. of the ratio of latency recorded as described in Material and methods. $*(\mathrm{P}<$ $0.05)$ designates significant differences versus control.

sure of MF did not change the activity of anti-oxidant enzymes including SOD and GPx. It is very interesting that MF exposure to rats induced lipid peroxidation with the raised MDA levels in every region we assayed. Lipid peroxidation is one of the major outcomes of free radical-mediated injury that directly damages membranes and generates a number of secondary products, both from fission and endocyclization of oxygenated fatty acids that possess neurotoxic activity. The unchanged antioxidant enzyme activity by MF exposure was found in this experiment. Considering the roles of anti-oxidation as enzymes in oxidative stress, this result leads us to hypothesis that where MF may act on may the non-enzymatic defense system such as glutathione. Under oxidative stress conditions, ROS are reduced by GSH with concomitant formation of the oxidized disulphide, GSSG. Although relatively resistant to 'spontaneous oxidation', GSH reacts rapidly and non-enzymatically with hydroxyl radical, and with $\mathrm{N}_{2} \mathrm{O}_{3}$ and peroxynitrite (Griffith, 1999). In addition to its action as a chemical antioxidant GSH also acts in the enzymatic first line antioxidant defense as a cofactor in GPx mediated reduction of peroxides, also resulting in GSSG formation. The overall reduction of reduced and oxidized glutathione by MF exposure has indicated that the chemical reaction requiring the electron transfer between ROS and glutathione or its derivatives may be influenced by MF stimuli.

In conclusion, the present study demonstrate that the exposure to MF for short or long term increase the thyroid hormone and alter the secretion of adrenal hormone in plasma. This effect leads to lipid peroxidation of whole brain mediated by oxidative stress with a decrease in the reduced and oxydized glutathione. From the results, we suggest that MF exposure may be an environmental stress via an effect on thyroid and adrenal hormone, and the consecutive oxidative stress.

\section{ACKNOWLEDGMENT}

This work was supported by Research Grant of Chung-Ang University (2006).

\section{REFERENCES}

Binhi, V. N., and Savin, A. V. (2002). Molecular gyroscopes and biological effects of weak extremely low-frequency magnetic fields. Phys. Rev. E. Stat. Nonlin. Soft Matter Phys. 65, 051912.

Bruner, L. J. and Harvey, J. R. (1998). Synchronization of pacemaker cell firing by weak ELF fields: simulation by a circuit model. Bioelectromagnetics. 19, 92-97.

Bruno, A. N., Fontella, F. U., Bonan, C. D., Barreto-Chaves, M. L., Dalmaz, C. and Sarkis, J. J. (2006). Activation of adenosine $A(1)$ receptors alters behavioral and biochemical parameters in hyperthyroid rats. Behav. Brain Res. 167, 287-294.

Duntas, L. H. (2005). Oxidants, antioxidants in physical exercise and relation to thyroid function. Horm. Metab. Res. 37, 572576.

Fernandez, V., Tapia, G., Varela, P., Romanque, P., Cartier-Ugarte, D. and Videla, L. A. (2006). Thyroid hormone-induced oxidative stress in rodents and humans: a comparative view and relation to redox regulation of gene expression. Comp. Biochem. Physiol. C. Toxicol. Pharmacol. 142, 231-239.

Hart, R. H., Kendall-Taylor, P., Crombie, A. and Perros, P. (2005). Early response to intravenous glucocorticoids for severe thyroid-associated ophthalmopathy predicts treatment outcome. J. Ocul. Pharmacol. Ther. 21, 328-336.

Huffman, L. J., Beighley, C. M., Frazer, D. G., McKinney, W. G and Porter, D. W. (2006). Increased susceptibility of the lungs of hyperthyroid rats to oxidant injury: specificity of effects. Toxicology. 225, 119-127.

Ishii, Y. and Tanizawa, H. (2006). Effects of soyasaponins on lipid peroxidation through the secretion of thyroid hormones. Biol. Pharm. Bull. 29, 1759-1763.

Keck, M. E., Welt, T., Post, A., Muller, M. B., Toschi, N., Wigger, A., Landgraf, R., Holsboer, F. and Engelmann, M. (2001). Neuroendocrine and behavioral effects of repetitive transcranial magnetic stimulation in a psychopathological animal model are suggestive of antidepressant-like effects. Neuropsychopharmacology. 24, 337-349.

Lai, H., Carino, M. A. and Ushijima, I. (1998). Acute exposure to a $60 \mathrm{~Hz}$ magnetic field affects rats' water-maze performance. Bioelectromagnetics. 19, 117-122.

Lakin, M. L., Miller, C. H., Stott, M. L. and Winters, W. D. (1981). Involvement of the pineal gland and melatonin in murine analgesia. Life Sci. 29, 2543-2551.

Langer, P., Foldes, O., Kvetnansky, R., Culman, J., Torda, T. and El Daher, F. (1983a). Pituitary-thyroid function during acute immobilization stress in rats. Exp. Clin. Endocrinol. 82, 51-60.

Langer, P., Foldes, O., Macho, L. and Kvetnansky, R. (1983b). Acute and transient activation of pituitary-thyroid axis during 
unforced restriction in rats: component of nonshivering thermogenesis in conscious animals? Horm. Res. 17, 27-35.

Langer, P., Vigas, M., Kvetnansky, R., Foldes, O. and Culman, J. (1983c). Immediate increase of thyroid hormone release during acute stress in rats: effect of biogenic amines rather than that of TSH? Acta. Endocrinol. (Copenh). 104, 443-449.

Levick, S., Fenning, A. and Brown, L. (2005). Increased calcium influx mediates increased cardiac stiffness in hyperthyroid rats. Cell Biochem. Biophys. 43, 53-60.

Mazure, C. M., Quinlan, D. M. and Bowers, M. B., Jr. (1997). Recent life stressors and biological markers in newly admitted psychotic patients. Biol. Psychiatry. 41, 865-870.

Ossenkopp, K. P. and Cain, D. P. (1988). Inhibitory effects of acute exposure to low-intensity $60-\mathrm{Hz}$ magnetic fields on electrically kindled seizures in rats. Brain Res. 442, 255-260.

Pang, C. S., Tsang, S. F. and Yang, J. C. (2001). Effects of melatonin, morphine and diazepam on formalin-induced nociception in mice. Life Sci. 68, 943-951.

Pantos, C., Malliopoulou, V., Mourouzis, I., Thempeyioti, A., Paizis, I., Dimopoulos, A., Saranteas, T., Xinaris, C. and Cokkinos, D. V. (2006). Hyperthyroid hearts display a phenotype of cardioprotection against ischemic stress: a possible involvement of heat shock protein 70. Horm. Metab. Res. 38, 308-313.

Senft, A., Dalton, T. and Shertzer, H. (2000). Determining glutathione and glutathione disulfide using the fluorescence probe o-phthalaldehyde. Anal. Biochem. 280, 80-86.

Sun, W., Yu, Y., Fu, Y., Chiang, H., Xie, H. and Lu, D. (2002). [Effects of power-frequency magnetic fields exposure on phosphorylation and enzymatic activity of stress-activated protein kinase and its upstream kinase]. Zhonghua Lao Dong. Wei Sheng Zhi Ye Bing Za Zhi. 20, 256-259.

Trimmel, M. and Schweiger, E. (1998). Effects of an ELF $(50 \mathrm{~Hz}$, $1 \mathrm{mT}$ ) electromagnetic field (EMF) on concentration in visual attention, perception and memory including effects of EMF sensitivity. Toxicol. Lett. 96-97, 377-382.

Varghese, S., Shameena, B. and Oommen, O. V. (2001). Thyroid hormones regulate lipid peroxidation and antioxidant enzyme activities in Anabas testudineus (Bloch). Comp. Biochem. Physiol. B. Biochem. Mol. Biol. 128, 165-171.

Venditti, P. and Di Meo, S. (2006). Thyroid hormone-induced oxidative stress. Cell Mol. Life Sci. 63, 414-434.

Walker, L. S., Garber, J., Smith, C. A., Van Slyke, D. A. and Claar, R. L. (2001). The relation of daily stressors to somatic and emotional symptoms in children with and without recurrent abdominal pain. J. Consult. Clin. Psychol. 69, 85-91.

Woldanska-Okonska, M. and Czernicki, J. (2003). [Influence of pulsating magnetic field used in magnet therapy and magnet stimulation on cortisol secretion in human]. Med. $\mathrm{Pr} .54,29$ 32. 